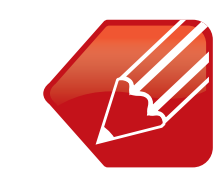

\title{
Rediseño y análisis de la inserción de los cursos introductorios de matemáticas, como un acercamiento lúdico a las matemáticas de primer semestre
}

Sara Giraldo y Lilian Paola Umbarila

Recibido: agosto 13 de 2012 Aprobado: octubre 05 de 2012

\begin{abstract}
Resumen
Este artículo publica los resultados obtenidos luego de realizar el proyecto de investigación basado en la recolección de experiencias entre docentes y estudiantes al desarrollar los cursos introductorios y que tuvieron como objetivo rediseñar las guías de estudio empleadas por los docentes. Los resultados sustentan teórica y didácticamente las actividades propuestas que justifican su inclusión como una materia previa para las asignaturas de matemáticas. El proyecto determina el impacto que tienen los cursos introductorios en el comportamiento actitudinal y académico de los estudiantes cuando se enfrentan a la vida académica en primer semestre.
\end{abstract}

Palabras clave: primer semestre, cursos introductorios de matemáticas, rendimiento académico, impacto, actitud, didáctica, enseñanza.

\section{Abstract}

This article publishes the results of the research project that aims to find the experiences that teachers and students have had in the development of introductory courses to redesign the guidelines used by teachers in these courses and theoretical and didactic support the proposed activities justify inclusion as a matter prior to the subjects of mathematics. The project determines the impact of introductory courses on attitudinal and academic performance of students when faced their academic life in the first sesmester.

Keywords: Introductory mathematics course, performance, impact, attitude, teaching, first semester.

1 Magister en Educación Matemática - Magister en Scientiae Matemáticas

2 Especialista en investigación y docencia Universitaria y candidato a Magister en Administración de Energías y Fuentes Renovables 


\section{INTRODUCCIÓN}

El proceso de enseñanza y aprendizaje de las matemáticas ha generado una amplia literatura alrededor de la práctica docente y del conocimiento o la forma como el estudiante concibe sus procesos internos. Tanto la educación básica como la media y la superior se han interesado por la relación que existe entre estos componentes y su impacto en la educación, por esto se busca que el estudiante tenga la oportunidad de conocer esta asignatura como una ciencia de vital importancia para su desarrollo mental y como una herramienta para desenvolverse en sus actividades cotidianas, a partir del entendimiento de que la matemática es una ciencia dinámica y cambiante.

Desde el año 1996, la Dirección de Primer Semestre de la Corporación Universitaria Minuto de Dios -UNIMINUTO lideró proyectos asociados con la deserción estudiantil en los primeros semestres, para lo cual ha realizado investigaciones y diseñado estrategias que disminuyan los índices de este fenómeno. Según las estadísticas proporcionadas por esta Dirección, en 2003 los índices mostraron una deserción de $28.19 \%$ y en el año 2008 de $23.92 \%$, lo cual muestra una disminución significativa del $4.27 \%$ en el abandono de las carreras por parte de los estudiantes que cursan los primeros semestres.

Cuando el estudiante llega a primer semestre de educación superior se encuentra con una matemática un poco más rígida y exigente. En el paso de la media a la universidad el estudiante asume roles diferentes en los que se exige mayor madurez mental y actitudinal. Las matemáticas introductorias de los primeros semestres requieren que aprenda o recuerde los procesos algebraicos un poco más rápido y esto hace que en algunos casos no se adapte, pierda la asignatura y en otros casos, incluso, el semestre.

UNIMINUTO preocupada por esta situación y con la colaboración del MEN (contrato No. 386 de 2007 suscrito entre el MEN y UNIMINUTO), llevó a cabo en el año 2008 el proyecto "Acompañamiento en primer semestre: prevención del abandono académico", dentro del cual se estableció la creación de un curso introductorio de matemáticas el cual tenía por objeto que el estudiante conociera las matemáticas desde una perspectiva lúdica, así como la importancia del uso de estas en su profesión y en su vida, a la vez que se adaptaba a la universidad. Los cursos introductorios se encuentran registrados en el numeral $12 \mathrm{del}$ código SNIES ${ }^{3}: 2829$ bajo el nombre de Implementa-

3 El SNIES, es el Sistema Nacional de Información de la Educación Superior y actúa como fuente de información, en relación con las instituciones y programas académicos aprobados por el Ministerio de Educación Nacional, consolida y suministra datos, estadísticas e indicadores ción de cursos recuperatorios dentro del mismo semestre académico.

Como mejora de esta estrategia se plantea el rediseño de las guías del curso introductorio, involucrando las competencias, objetivos y, si es necesario, modificar sus contenidos a partir de las experiencias de los docentes. Además de lo anterior, se busca analizar la incidencia de la introducción de este curso en la perdida académica de los estudiantes de primer semestre que ven matemáticas, desde su actitud y desempeño.

\section{METODOLOGÍA}

La metodología planteada se divide en dos actividades de acuerdo con los objetivos que se persiguen cumplir durante esta investigación. Estas son, el rediseño de las guías sustentadas teóricamente y la medición del impacto que genera el curso introductorio de matemáticas.

\section{Rediseño de las guías}

El rediseño de las guías se realizó de acuerdo con un conjunto de encuestas que se aplicaron tanto a los estudiantes como a los docentes que habían participado en experiencias pasadas de estos cursos introductorios. Según los resultados se encontró que era necesario incluir una guía preliminar al tema que permita al estudiante, en forma contextual, introducirse en cada uno de los contenidos a trabajar durante las sesiones del curso.

Además de lo anterior, el rediseño incluye ejercicios de repetición, ya que estos facilitan la mecanización y apropiación de los algoritmos. Dentro de los ejercicios mecanizables se presentan otro tipo de actividades que conllevan a la resolución de problemas, las cuales juegan un papel importante puesto que permiten generar y promover la participación activa y colectiva a nivel intelectual, que a través de la experimentación y el análisis de las circunstancias, culmina con la formulación y generalización de hechos desde una óptica científica con aplicación en diferentes áreas del conocimiento.

Los docentes también manifestaron que hacía falta la formalidad matemática en los contenidos y una introducción teórica a los mismos, además de incluir cada tema en un contexto histórico como una forma lúdica para acercar a los estudiantes con cada uno de los temas tratados, ya que esta permite ampliar el panorama del estudiante respecto a las circunstancias sobre las cuales se generó la matemática como ciencia, específicamente en respuesta a las necesidades de la humanidad, tal y como sucedió con la geometría. 
Luego se eligieron los temas centrales que iban a formar parte de los contenidos del curso introductorio, a través de seis guías.

\section{Un mundo sin matemáticas}

La guía pretende que los estudiantes entiendan la importancia de las matemáticas en el contexto socio cultural, en el desarrollo industrial y tecnológico, en el programa que escogió para estudiar y en su propia vida. Además busca que los estudiantes se integren e interactúen para que se vayan iniciando en el ambiente universitario.

En esta guía, los estudiantes deben reconocer:

a) El papel que juegan las matemáticas en la sociedad, comprendiendo y valorando su evolución y el impacto que ha generado en el desarrollo, mejorando el nivel de vida de las naciones.

b) Las aplicaciones que tienen las matemáticas desde actividades cotidianas muy sencillas hasta aplicaciones en diferentes campos como la medicina, la geografía, ingeniería, sistemas, redes, etc.

Para lo anterior, se cuenta con una actividad de reconocimiento que usa la criptografía, ésta permite que los estudiantes cifren sus nombres o alguna frase importante para la universidad. En esta actividad lúdica se pueden involucrar oraciones propias de la Institución para que los estudiantes reconozcan el lugar en donde van a estudiar y se integren con sus compañeros.

Luego se propone un ejercicio en donde ellos imaginan un mundo sin matemáticas (proyecto de primer año propuesto por UNIMINUTO y con el que ganó el concurso con el MEN). Finalmente se proponen otras lecturas para hacer que el estudiante analice que las matemáticas no son una ciencia aislada, sino que permite alcanzar los objetivos propuestos anteriormente.

\section{Un mundo de números}

A través de diferentes situaciones se hace que el estudiante relacione los sistemas numéricos con su entorno a la vez que se introducen los formalismos propios de cada conjunto, las diferentes formas de representación y sus propiedades. La intención de la guía no es enseñar a operar los números reales, sino presentar los sistemas numéricos y sus axiomas, y guiar al estudiante para que vaya analizando subconjuntos, el infinito de cada uno y las relaciones entre números que no se pueden dar debido a las propiedades que poseen.

Es importante que el estudiante refuerce sus conocimientos en este tema, ya que como lo afirma Lestón (2006), el aprendizaje erróneo de los sistemas numéricos puede llevar a la escasa compresión de otros conceptos, "como la idea de límite, variación, derivada, función continua y otros concernientes al campo del análisis matemático, que se basan en los anteriores". (p. 35)

Con esta guía se espera que el trabajo con los sistemas numéricos de los reales, desde sus diferentes tipos de representaciones y formalismos, permita que el estudiante recuerde, analice y comprenda estos objetos matemáticos con mayor confianza, logrando así que tenga unas bases más estructuradas y elaboradas para un correcto aprendizaje del cálculo.

Aquí se hace referencia a las diferentes formas de representar los conjuntos y la importancia que tienen en los diferentes contextos disciplinares y cotidianos. Estos aspectos son considerados por Bruno (1997) como las tres dimensiones: abstracta, recta y contextual. La dimensión abstracta hace referencia a los sistemas numéricos como estructuras matemáticas y las formas de escritura de los números. La dimensión recta alude a su representación geométrica, utilizando los puntos sobre ella y los vectores para identificar los números reales. La dimensión contextual se encuentra en las utilidades y usos de los números. Estas tres dimensiones y las diferentes transferencias entre estas permitirán que el estudiante pueda relacionar la matemática abstracta aprendida con lo concreto, a la vez que se logra el objetivo lúdico del curso.

\section{Los números se transforman}

Esta guía tiene por objeto que los estudiantes recuerden las diferentes operaciones que se pueden realizar con los números reales de acuerdo con las propiedades que tienen cada uno de ellos y los axiomas aprendidos en la guía anterior. Se plantean actividades que relacionan al estudiante con los contenidos propios del tema de estudio y del programa que pretende estudiar, además, se involucran ejercicios que hagan referencia al contexto universitario en el que se desenvolverá en el siguiente semestre.

Durante el contenido se da la relevancia a los algoritmos aritméticos que conducen a efecturar las operaciones con los números reales, ya que estas son la base para la comprensión de las operaciones que se pueden realizar con las expresiones algebraicas. Estos algoritmos son importantes para los conocimientos previos y competencias que debe tener un estudiante cuando ingresa a los primeros niveles universitarios de matemáticas.

De acuerdo al estudio realizado en la Universidad Panamericana, Cortés (2009) encontró que los estudiantes que ingresaban a primer semestre aplicaban intuitivamente las propiedades de la suma, la multiplicación y la división de los números racionales y que, en general, no reconocían las propiedades de suma y 
multiplicación de los números reales. Esto no se aleja del escenario que se vive en UNIMINUTO, ya que, aunque no existe un estudio que clasifique el nivel de matemáticas que alcanzan los estudiantes que ingresan, las pérdidas académicas de primer semestre sí lo deja entrever. No obstante, como se mencionó en los preliminares de la investigación, si bien el curso introductorio no pretende nivelar a los estudiantes sí es una opción para recordar los conocimientos aprendidos en la educación media.

Los contenidos planteados se muestran a través de actividades lúdicas, como la regleta inglesa 1 que permite realizar operaciones con los números racionales y laberintos donde se puede dar la mecanización de operaciones para conseguir la apropiación de los algoritmos.

\section{El número en forma general}

La temática permite que el estudiante generalice situaciones matemáticas y en contexto utilizando el lenguaje algebraico. Debe ser capaz de utilizar la letra para describir diferentes situaciones y traducir expresiones algebraicas a un escenario concreto. Esta guía tiene un conjunto de ejercicios que comprenden operaciones muy sencillas con expresiones algebraicas que se deducen de actividades lúdicas.

Es importante mencionar que en la educación media, la transición de la aritmética al álgebra genera ciertas dificultades en los estudiantes que se van a ver reflejadas en la incapacidad para entender un problema y modelarlo. Muchos consideran el álgebra desde la noción operacional, hacen uso sus algoritmos y utilizan las letras como objetos sin significados, lo que conlleva a los frecuentes errores y dificultades. Dentro de estas dificultades se encuentran (Mata, 2009):

- Realizar transformaciones algebraicas aplicando propiedades de las operaciones en $\mathrm{R}$ necesarias para escribir la igualdad correcta.

- Utilizar correctamente el lenguaje algebraico.

- Efectuar abstracciones y generalizaciones.

La mejor forma de definir el lenguaje algebraico puede ser la dada por Paralea (1999) al entenderla como:

... lenguaje en este acercamiento semiótico, se entiende como un sistema de representación que se ocupa del significado de las escrituras algebraicas, además de considerar el carácter instrumental de signos del álgebra... En resumen el signo algebraico será considerado como parte de un "portavariable" (hace las veces de), constituye una herramienta de modelización de sistemas no algebraicos (numérico y geométrico, etc) y por otra, funciona como un signo de sí mismo, es decir como un instrumento específico de la actividad. (p. XI)
En la guía la variable se usa tanto como un objeto de la actividad en sí misma, cuando se realizan operaciones con él y, como un portavariable, en este sentido la letra se puede considerar como una variable que puede adquirir diferentes significados de acuerdo al contexto y al sentido que se le este dando. Se utilizan contextos geométricos y numéricos teniendo en cuenta la relevancia que tienen los sistemas de representación.

El álgebra constituye el lenguaje matemático, por tanto se hace necesario el uso de los símbolos en forma adecuada lo que lleva a que la guía esté orientada a representaciones de situaciones muy sencillas y geométricas que permitan al estudiante evidenciar la utilidad del modelado para representar los diferentes contextos propuestos.

\section{De la recta al espacio}

La geometría juega un rol importante dentro de los programas de ingeniería. Esta permite modelar situaciones tales como distribuciones de planta, mediciones y cálculos acertados en construcciones, etc.

Esta guía tiene como objetivo el análisis de las tres dimensiones, las nociones de punto, recta, plano y espacio. Se plantean lecturas que posibilitan al estudiante la ubicación en una recta, en un plano y en el espacio, y el reconocimiento de figuras geométricas planas y sólidos redondos y regulares. Con lo anterior el estudiante puede usar representaciones geométricas, el razonamiento espacial y el modelado geométrico para resolver problemas.

Los contenidos encontrados aquí no pretenden desarrollar niveles de aprendizaje complejos, tales como demostraciones, lo que se pretende es tomar algo de la geometría de sólidos, de la geometría euclidiana y del espacio y plano coordenado, para recordar algunas temáticas, es decir, lo que se busca es desarrollar los niveles 0,1 y 2 de Van Hiele, mostrados en la Figura 1.

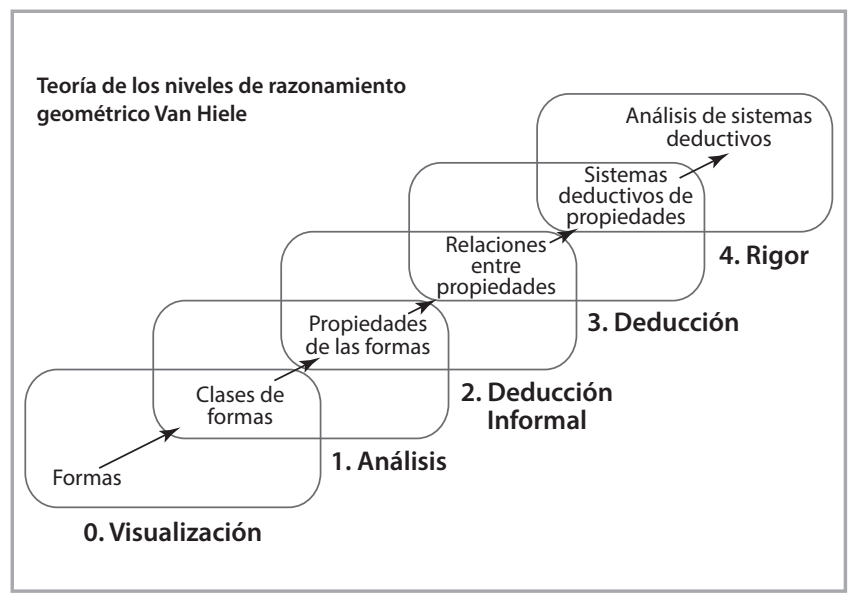

Figura 1. Teoría de los niveles de razonamiento geométrico de Van Hiele. Adaptado de Godino y Ruiz (2004), p. 298. 
Estos niveles permiten comprender y orientar el pensamiento geométrico de los estudiantes. Para ello se proponen cinco niveles jerárquicos que se obtienen como producto de realizar diferentes tareas en las que se va aumentando el grado de dificultad.

Estos niveles se alcanzan independientemente de la edad y son secuenciales, esto quiere decir que para lograr alcanzar el nivel 2 se necesita haber pasado primero el nivel 0 y luego el nivel 1 .

En el nivel 0 los objetos son formas y se conciben según su apariencia, en este caso el producto de pensamiento que se espera son clases o agrupaciones de forma según su apariencia.

En el nivel 1 los objetos son clases de formas más no formas individuales, se espera que el estudiante reconozca las propiedades de las formas.

En el nivel 2 los objetos de pensamiento son las propiedades de las formas y se pretende que el estudiante establezca relaciones entre las propiedades de los objetos geométricos, las propiedades comienzan a centrase en argumentos lógicos y se pueden realizar demostraciones de tipo intuitivo mas no se alcanza un sistema deductivo formal.

En el nivel 3 los objetos de pensamiento son relaciones entre las propiedades de los objetos geométricos. Se hacen demostraciones de tipo deductivo a partir de axiomas y teoremas. Los productos esperados son sistemas axiomáticos para la geometría.

En el nivel 4 los objetos de pensamiento son sistemas axiomáticos para la geometría. El nivel máximo donde se presta atención a los propios sistemas axiomáticos mas no a las deducciones. Es propio de cursos universitarios especializados. Los productos son comparaciones y contrastes entre los diferentes sistemas axiomáticos de geometría.

Como el objetivo del curso no es nivelar al estudiante, como ya se ha mencionado, solo se pretende llegar hasta el nivel 2 , reconocimiento de las figuras y cuerpos geométricos y clasificación de acuerdo con sus propiedades; aunque, según Van Hiele, el tercer nivel se debió alcanzar en la formación media.

Para el desarrollo de los contenidos se usa el tangram con el objeto de que el estudiante calcule áreas y perímetros de las figuras. También se les pide la caracterización de poliedros a través de construcciones que ellos mismos hacen en clase.

\section{Ecuaciones: álgebra con papas}

Esta guía se lleva a cabo en línea con el software
“Álgebra con papas", que consta de actividades elaboradas con el recurso educativo en línea hot potatoes ${ }^{4}$ para el desarrollo de contenidos aritméticos y algebraicos. En esta guía no se realizaron modificaciones de fondo, solo de forma. El programa se presta para que a medida que las actividades se vayan desarrollando, también lo hagan los contenidos que muestran los diferentes algoritmos para resolver las ecuaciones de diferente grado. Este programa cuenta con la ventaja que los estudiantes pueden continuar desde su casa usándolo, ya que se encuentra en Internet.

\section{Mediciones de los cursos Introductorios}

Para realizar la investigación del impacto que generan los cursos introductorios de matemáticas en el rendimiento académico de los estudiantes y determinar los resultados que generan estos dentro del Departamento de Ciencias Básicas de UNIMINUTO, se llevó a cabo un estudio que incluye el análisis de la efectividad de los cursos a través de la eficiencia y la eficacia de los mismos.

La eficiencia es un indicador de gestión del proceso. Este indicador abarca la cobertura, costos y asistencia.

Para lograr este objetivo, se realizaron los siguientes procedimientos:

a. Se tomaron dos muestras de estudiantes, una de primer semestre y otra de segundo con el objeto de analizar el aspecto: "que tanto les gusta el curso introductorio de matemáticas" de acuerdo con la vivencia que han tenido. Después se compararon los dos resultados, teniendo en cuenta que el primer grupo estaba cursando primer semestre y podía proporcionar una visión más objetiva, dada la experiencia adquirida en UNIMINUTO y el cual enfatiza en la adaptación a la vida universitaria; característica que influye bastante en la conducta y rendimiento del estudiante. Al segundo grupo se le dio una perspectiva más amplia, ya que sus integrantes terminaron primer semestre y podían realizar comparaciones más acertadas acerca de los beneficios o desventajas del curso.

b. Se realizó una entrevista a un estudiante que hizo el primer curso introductorio.

c. Se determinó el capital invertido por semestre. Este aspecto abarca la nómina de los docentes, gastos en material didáctico proporcionado a los estudiantes y gastos de infraestructura.

La eficacia es un indicador de resultados y muestra directamente el impacto del curso en el rendimiento

4 Hot Potatoes es un software educativo utilizado para realizar actividades lúdicas en diferentes contextos de la enseñanza. 
académico y en su comportamiento actitudinal durante el primer semestre.

Este indicador se evalúo a través de:

a. Comparación entre las calificaciones de estudiantes de primer semestre que han hecho el curso y estudiantes de semestres anteriores que no asistieron al mismo.

b. Comparación entre las inquietudes acerca de la metodología empleada y las propuestas de los estudiantes de primer semestre cuando se ha llevado a cabo el curso y en ausencia de él.

La selección del tamaño de la muestra, así como los instrumentos diseñados para indagar acerca del fenómeno "qué tanto les gusta el curso introductorio", representan una investigación de tipo cualitativo. Dentro de esta, se emplea la encuesta para cuestionar al estudiante sobre la experiencia que ha vivido en el curso introductorio; sin embargo, no se pretende manipular los datos para comprobar si sirve o no, solamente se busca analizar la influencia que éste ha tenido en su comportamiento durante su vida universitaria y en su rendimiento académico en matemáticas.

Según Sandoval (1996) la metodología cualitativa que conlleva a la descripción de la experiencia humana vivida, sin tener en cuenta las causas que lo originan, corresponde a una metodología fenomenológica. Para el caso, en este estudio no se expone el por qué al estudiante le atrae o no el curso, solo se pretende poner de manifiesto su posición ante un suceso que pudo o no haber repercutido en su rendimiento y actitud en el inicio y transcurso del primer semestre en la universidad.

Con respecto al tamaño de la muestra, Mayan María (2001) establece que en la investigación cualitativa, ésta se elige intencionalmente, al respecto afirma:

El investigador elige individuo y contextos al preguntarse: ¿Quién puede darme mayor y mejor información acerca de mi tópico?

¿En qué contexto seré capaz de reunir la mayor y mejor información acerca de mi tópico?

Entonces se seleccionan individuos y contextos desde los cuales puede aprenderse mucho acerca del fenómeno. (p.10)

De acuerdo con lo anterior, para este estudio se propuso tomar una muestra de 15 estudiantes de segundo semestre de Ingeniería y 15 de Ciencias Empresariales, ya que estos cursos están dirigidos a estudiantes que van a cursar primer semestre en cada una de estas facultades. En la misma cantidad se hizo el estudio para los estudiantes de primer semestre de acuerdo con la metodología planteada en las mediciones de los cursos introductorios.

\section{RESULTADOS}

\section{Análisis de la eficiencia}

a. Encuesta a los estudiantes

Las respuestas obtenidas de la encuesta fueron positivas para la investigación, como lo muestran las tablas 1 y 2 .

Tabla 1. Resultados obtenidos en la aplicación de la encuesta a la muestra 1

\begin{tabular}{|c|c|c|c|}
\hline PREGUNTA & RESPUESTA & FRECUENCIA & $\begin{array}{l}\text { FRECUENCIA } \\
\text { PORCENTUAL }\end{array}$ \\
\hline \multirow{2}{*}{$\begin{array}{l}\text { 1. ¿Usted realizó el curso introductorio } \\
\text { de matemáticas? }\end{array}$} & $\mathrm{Si}$ & 25 & 83 \\
\hline & No & 5 & 17 \\
\hline \multirow{2}{*}{$\begin{array}{l}\text { 2. ¿Considera que el haber hecho el curso fue } \\
\text { importante para ubicarse dentro de la universidad } \\
\text { antes de iniciar el primer semestre? }\end{array}$} & $\mathrm{Si}$ & 19 & 63 \\
\hline & No & 6 & 20 \\
\hline \multirow{4}{*}{$\begin{array}{l}\text { 3. ¿Cómo ha influido el curso en su rendimiento } \\
\text { académico para el curso de precálculo o } \\
\text { matemáticas I? }\end{array}$} & No me sirvió & 5 & 17 \\
\hline & $\begin{array}{l}\text { Me permitió dar un conocimiento general } \\
\text { como introducción }\end{array}$ & 12 & 40 \\
\hline & $\begin{array}{l}\text { Me ayudó a prepararme para afrontar responsabi- } \\
\text { lidades frente a mi rendimiento académico. }\end{array}$ & 3 & 10 \\
\hline & $\begin{array}{l}\text { Me facilitó el aprendizaje de las } \\
\text { matemáticas de primer semestre }\end{array}$ & 5 & 17 \\
\hline \multirow{2}{*}{$\begin{array}{l}\text { 4. En caso de que no hubiera realizado el curso, } \\
\text { ¿considera que le hizo falta un espacio de } \\
\text { adaptación a la universidad? }\end{array}$} & $\mathrm{Si}$ & 4 & 13 \\
\hline & No & 1 & 3 \\
\hline \multirow{2}{*}{$\begin{array}{l}\text { 5. ¿Cree usted que es necesario usar un espacio para } \\
\text { realizar una introducción a las matemáticas antes } \\
\text { de cursar precálculo o matemáticas I? }\end{array}$} & $\mathrm{Si}$ & 4 & 13 \\
\hline & No & 1 & 3 \\
\hline
\end{tabular}

Tabla 1. Resultados de la encuesta a estudiantes de primer semestre de los diferentes programas ofertados por las facultades de Ingeniería y Ciencias Empresariales de la Sede Principal de UNIMINUTO (2011 - I). Por: autoras, 2011 
Tabla 2. Resultados obtenidos en la aplicación de la encuesta a la muestra 2

\begin{tabular}{|c|c|c|c|}
\hline PREGUNTA & RESPUESTA & FRECUENCIA & $\begin{array}{l}\text { FRECUENCIA } \\
\text { PORCENTUAL }\end{array}$ \\
\hline \multirow{2}{*}{$\begin{array}{l}\text { 1. ¿Usted realizó el curso introductorio } \\
\text { de matemáticas? }\end{array}$} & $\mathrm{Si}$ & 23 & 77 \\
\hline & No & 7 & 23 \\
\hline \multirow{2}{*}{$\begin{array}{l}\text { 2. ¿Considera que el haber hecho el curso fue impor- } \\
\text { tante para ubicarse dentro de la universidad antes } \\
\text { de iniciar el primer semestre? }\end{array}$} & $\mathrm{Si}$ & 20 & 67 \\
\hline & No & 3 & 10 \\
\hline \multirow{4}{*}{$\begin{array}{l}\text { 3. ¿Cómo ha influido el curso en su rendimien- } \\
\text { to académico para el curso de precálculo o } \\
\text { matemáticas I? }\end{array}$} & No me sirvió & 3 & 10 \\
\hline & $\begin{array}{l}\text { Me permitió dar un conocimiento general } \\
\text { como introducción }\end{array}$ & 8 & 27 \\
\hline & $\begin{array}{l}\text { Me ayudó a prepararme para afrontar responsabi- } \\
\text { lidades frente a mi rendimiento académico. }\end{array}$ & 9 & 30 \\
\hline & $\begin{array}{l}\text { Me facilitó el aprendizaje de las } \\
\text { matemáticas de primer semestre }\end{array}$ & 3 & 10 \\
\hline \multirow{2}{*}{$\begin{array}{l}\text { 4. En caso de que no hubiera realizado el curso, } \\
\text { ¿considera que le hizo falta un espacio de adap- } \\
\text { tación a la universidad? }\end{array}$} & $\mathrm{Si}$ & 6 & 20 \\
\hline & No & 1 & 3 \\
\hline \multirow{2}{*}{$\begin{array}{l}\text { 5. ¿Cree usted que es necesario usar un espacio para } \\
\text { realizar una introducción a las matemáticas antes } \\
\text { de cursar precálculo o matemáticas I? }\end{array}$} & $\mathrm{Si}$ & 7 & 23 \\
\hline & No & 0 & 0 \\
\hline
\end{tabular}

Tabla 2. Resultados de la encuesta a estudiantes de segundo semestre de los diferentes programas ofertados por las facultades de Ingeniería y Ciencias Empresariales de la Sede Principal de UNIMINUTO (2011 - I). Por: autoras, 2011

b. La entrevista a profundidad reveló que el estudiante se encontraba satisfecho con los contenidos vistos durante el curso, lo cual le permitió afrontar los miedos que traía por ser una persona mayor, posibilitándole integrarse y adaptarse mejor a la vida universitaria. Las respuestas no se muestran aquí ya que se encuentran consignadas en el documento que respalda la investigación.

\section{c. Costos del capital invertido por semestre}

De acuerdo con los datos proporcionados por la asistente de Ciencias Básicas, responsable del manejo del centro de costos del Departamento, se establecieron los costos que se generaron por la implementación del curso, los cuales se indican en la Tabla 3 para el primer semestre de 2011.

Tabla 3. Relación de costos

\section{DESCRIPCIÓN}

1. Valor promedio de la hora de nómina de docentes de tiempo completo y medio tiempo

2. Se dictaron 47 cursos de 20 horas cada uno

3. Costo de 45.275 copias del material suministrado a los estudiantes

4. Costo promedio debido a la jornada horaria usada (mañana, tarde y noche) de un salón para máximo 45 personas

5. Costo total de los 47 salones ocupados durante 4 días

6. Costo de uso de los laboratorios de informática (en promedio la hora a $\$ 13.089,33$ )

COSTO

$\$ 9.000$

$\$ 8.460 .000$

$\$ 2.263 .750$

$\$ 13.030,67$

$\$ 7.349 .297,88$

$\$ 1.230 .397,33$

Total por semestre

19.303.444.9

La Tabla 3. Evidencia que para la Institución la inversión debido al costo por estudiante, suponiendo que hay 40 estudiantes en cada curso, es de $\$ 10.267,79$, lo que sugiere un costo bajo comparándolo con los ingresos que se dejarían de percibir si el estudiante desertara.

\section{Resultados de la eficacia}

Asistieron al curso intersemestral del primer semestre de 2011, un total 431 estudiantes, de los cuales aprobaron 398, lo que indica que de los asistentes al curso solo lo reprobaron el $7.66 \%$ aproximadamente.

\section{ANÁLISIS DE RESULTADOS}

\section{Análisis de la eficiencia de los cursos introductorios de matemáticas}

Dentro de la muestra seleccionada se observa que los estudiantes que ingresaron a la universidad en el primer semestre de 2011 asistieron en su mayoría al curso introductorio con relación al número del semestre anterior, con una diferencia del $6 \%$.

En tanto a la utilidad de los cursos para adaptar al estudiante a la vida universitaria, del grupo que ingresó a primer semestre de 2011, se evidenció que del $83 \%$ que estuvo en el curso, el $63 \%$ contestó que sí, mientras que en el curso que ingresó antes se observó que del $77 \%$, el $67 \%$ apreciaron que el curso les sirvió para integrarse en primer semestre. Lo anterior implica que los de segundo semestre, que obviamente finalizaron su primer semestre e inclusive puede haber repitentes, tienen una perspectiva más amplia acerca de las utilidades que les brindó el curso universitario. Lo mismo se evidencia en la encuesta a profundidad, donde el estudiante que ingresó semestres atrás se mostró muy interesado por el conocimiento aprendido del curso y por la ganancia en todos los aspectos que generó en él dicho curso.

En cuanto a la tercera pregunta, se observa que de la muestra poblacional que realizó más recientemente 
el curso (primer semestre de 2011), el 17\% de los estudiantes indicó que el curso introductorio no influenció en su rendimiento académico, pero analizando el grupo que ingresó un semestre atrás, los de segundo semestre, tan solo el $10 \%$ se sintió identificado con esta respuesta. En la misma pregunta el $40 \%$ de los estudiantes de 2011-I indicaron que el curso les había servido como inducción general al curso de precálculo o matemática I, mientras que en el otro grupo se manifiesta un patrón interesante indicando porcentajes muy parecidos en las respuestas de "me permitió dar un conocimiento general como introducción" y "me ayudó a prepararme para afrontar responsabilidades frente a mi rendimiento académico".

Lo anterior indica que el curso introductorio no solo induce al estudiante en sus conocimientos matemáticos de primer semestre sino que también modifica su comportamiento y actitud en primer semestre, transformándolos en positivos tanto en la parte cognitiva como en la actitudinal.

Aunque hay estudiantes que piensan que realmente el curso no les sirvió ni cognitivamente ni en su forma de afrontar responsabilidades, hay un porcentaje considerable de un tercer grupo, conformado por los que no llevaron a cabo el curso, y quienes desearían que existieran espacios como estos para prepararlos para la vida universitaria, integrándolos y fortaleciendo sus conocimientos. En el grupo que tuvo el curso en el segundo semestre, $13 \%$ del $17 \%$ que no asistieron y en el segundo grupo el $20 \%$ del $23 \%$ manifestaron que deberían existir este tipo de espacios para la preparación de los estudiantes para ser más responsables y para madurar frente a sus compromisos. Este porcentaje aumenta en los dos grupos cuando se hace referencia a las ventajas que trae frente a los contenidos temáticos impartidos en los primeros cursos de matemáticas.

Lo anterior se corrobora con las observaciones hechas por los estudiantes en los espacios en blanco destinados para ello y en la entrevista que se ha hecho a profundidad, donde el estudiante manifestó que el curso no solo le dio las bases y los conocimientos que hacía 25 años no recordaba, sino que además le permitió integrarse con sus compañeros, ya que él tenía temores debido a su edad.

En cuanto al análisis económico, el Departamento estaría generando sobre costos al apoyar la realización de los cursos, pero si se realiza un análisis por unidad (estudiante), se estaría logrando una ganancia frente al costo que les genera a ellos un curso intersemestral o para la universidad la deserción de un estudiante.

Análisis de la eficacia del curso Introductorio En cuanto al número de inquietudes, se evidenció que en el Departamento de Ciencias Básicas, tan sólo cinco quejas formales presentaron los estudiantes de primer semestre, esto se da debido a que el estudiante ya ha cambiado previamente su mentalidad, donde en el colegio el profesor jugaba un papel sobre protector. Los cursos tienen por objeto que el estudiante se responsabilice de su propio aprendizaje, realizando las tareas y generando un aprendizaje más autónomo.

\section{CONCLUSIONES}

Como se esperaba, en un porcentaje significativo, los estudiantes demostraron que a través de un curso introductorio adquieren una madurez en cuanto a su incursión a las matemáticas de primer semestre y a la responsabilidad que ellos deben afrontar académicamente, al ser más autónomos y comprometidos con su propio aprendizaje.

En los resultados se evidencia que el grupo encuestado que inicia su primer semestre se ve más reacio a identificar la importancia que tiene el curso introductorio, mientras que los que son de segundo semestre se sintieron más satisfechos frente a los beneficios que les dejó el introductorio de matemáticas. Esto puede ser a causa de dos factores. El primero, la inmadurez que quizás presentan los estudiantes de primer semestre o, el segundo, la influencia que ejerce el docente tanto en el introductorio como en el primer curso de precálculo, lo que puede hacer o no que los estudiantes cambien su actitud hacia las matemáticas.

Los costos que generan los cursos introductorios comparados con los que se pueden originar por la deserción de un estudiante, o para que él mismo realice un curso intersemestral, son mucho menores.

El número de quejas disminuye cuando los introductorios se ofrecen, ya que estos permiten que el estudiante se separe de las actitudes que se presentan en la educación media, haciendo que sea consciente de su propio aprendizaje y afronte sus responsabilidades con mayor compromiso.

\section{REFERENCIAS BIBLIOGRÁFICAS}

1. Agalde, A. (2009). Rendimiento en cursos introductorios de matemática. Principales errores detectados y posibles soluciones. Costa Rica: Escuela de matemáticas, Universidad Nacional de Costa Rica.

2. Angulo, M. (2009). Sistema para la prevención y análisis de deserción en las instituciones de Educación superior- Spadies. Bogotá: Ministerio de Educación Nacional. 
3. Bruno, A. (marzo de 1997). La enseñanza de los números negativos, aportaciones de una investigación. Revista didáctica de las matemáticas. 29: 5-18.

4. Cortes, H. (2009). Estrategia didáctica para la enseñanza de la matemática fundamental. Revista Dialéctica. 29: 20-29.

5. Díaz, C. (2008) Modelo conceptual para la deserción estudiantil universitaria chilena. Estudios Pedagógicos (34) 2: 65-86 Recuperado de http//:www.scielo.cl/scielo.php?pi$\mathrm{d}=\mathrm{s}$ 0718-07052008000200004\&script=sci_arttext. Recuperado marzo de 2010.

6. Godino, J y Ruiz, F. (2004). Didática de las matemáticas para los maestros. España: Departamento de Didáctica de la Matemática Facultad de Ciencias de la Educación Universidad de Granada.

7. Lestón, P. (2006). Ideas de los alumnos de Escuela Media sobre el infinito de los conjuntos numéricos. Revista Premisa. 29: 35-42.

8. Martínez, B. (2008). Informe de Gestión (julio de 2006- Julio de 2008). Dirección de Primer semestre UNIMINUTO Sede Principal. Bogotá: UNIMINUTO.

9. Mata, L. et al (28 a 30 de octubre de 2009). II Jornada de Enseñanza e Investigación Educativa en el campo de las Ciencias Exactas y $\mathrm{Na}$ turales. Actas, II.. Departamento de Ciencias Exactas y Naturales. FaHCE (UNLP). Argentina.
10. Mayan, M. (2001). Una introducción a los métodos cualitativos: módulo de entrenamiento para estudiantes y profesionales. Cisneros, C (Trad). Estados Unidos: Qual Intitute Press. International Institute for Qualitative metodology.

11. MEN, (2009). Diagnóstico de la deserción estudiantil en Colombia. http://www.universia. net.co/index2.php?option=com_conten\&do_ $\mathrm{pdf}=1 \& i d=13058$

12. Paralea, M. (diciembre de 1999). La adquisición del lenguaje algebraico: reflexiones de una investigación. Revista didáctica de las matemáticas. 40: 3-28.

13. Reyes, A. (1998). Análisis de la efectividad del curso introductorio en la asignatura matemática para la facultad de industrial. Revista cubana de educación superior, ( 8) 23-32.

14. Sandoval, C (1996). Investigación cualitativa. Bogotá: Instituto Colombiano para El Fomento de la Educación Superior- ICFES.

15. UNIMINUTO. (2009) Resultados Encuesta Seguimiento Académico Ciencias Básicas 2009-I. Bogotá: Autor 